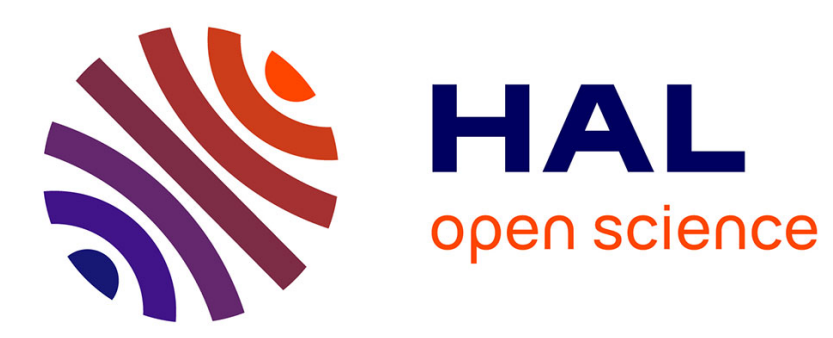

\title{
Sur la possibilité d'utilisation de sources phosphorescentes excitées par un élément radioactif pour la calibration en campagne
}

\author{
J.M. Vreux, G. Marette
}

\section{- To cite this version:}

J.M. Vreux, G. Marette. Sur la possibilité d'utilisation de sources phosphorescentes excitées par un élément radioactif pour la calibration en campagne. Revue de Physique Appliquée, 1969, 4 (3), pp.421-422. 10.1051/rphysap:0196900403042100 . jpa-00243306

\section{HAL Id: jpa-00243306 https://hal.science/jpa-00243306}

Submitted on 1 Jan 1969

HAL is a multi-disciplinary open access archive for the deposit and dissemination of scientific research documents, whether they are published or not. The documents may come from teaching and research institutions in France or abroad, or from public or private research centers.
L'archive ouverte pluridisciplinaire HAL, est destinée au dépôt et à la diffusion de documents scientifiques de niveau recherche, publiés ou non, émanant des établissements d'enseignement et de recherche français ou étrangers, des laboratoires publics ou privés. 


\title{
SUR LA POSSIBILITÉ D’UTILISATION DE SOURGES PHOSPHORESGENTES EXGITÉES PAR UN ÉLÉMENT RADIOAGTIF POUR LA GALIBRATION EN GAMPAGNE
}

\author{
Par J. M. VREUX, \\ Aspirant F.N.R.S., \\ et G. MARETTE, \\ Institut d'Astrophysique de l'Université de Liège, Cointe-Sclessin (Belgique). \\ (Reçu le 17 mars 1969, vévisé le 9 juin 1969.)
}

\begin{abstract}
Résumé. - Après avoir remarqué la grande stabilité de l'émission de certaines sources phosphorescentes, nous avons réalisé un dispositif fort compact permettant leur utilisation pour la calibration des plaques et films photographiques en campagne.

Abstract. - Having observed the high stability of emission of certain phosphor sources, a very compact device has been fabricated to use them for calibration purposes of photographic plates and films during campaigns.
\end{abstract}

Les sources phosphorescentes excitées par un élément radioactif sont largement utilisées par les chercheurs qui se trouvent dans l'obligation de transporter un étalon de brillance hors des laboratoires. Toutefois, il a été signalé par certains expérimentateurs [1] qu'une illumination extérieure exerce une influence sur leur émission. Désirant employer des sources de ce type pour contrôler pendant des campagnes d'observation la calibration de films et de photomètres, nous avons jugé indispensable de vérifier si celles que nous comptons utiliser comportent le même défaut.

Pour ces essais, nous disposons de deux sources phosphorescentes à excitation par particules $\beta$ :

- Une source "bleue »: maximum d'émission à $(4540 \pm 25) \AA$, largeur à mi-hauteur : (650 $\pm 20) \AA$.

- Une source " verte »: maximum d'émission à $(5270 \pm 25) \AA$, largeur à mi-hauteur : $\quad(650$ $\pm 20) \AA$. L'irradiance à $5577 \AA$ de cette dernière source vaut $2,8 \times 10^{5}$ photons/s, $\mathrm{cm}^{2}, 10 \AA$ à une distance de $50 \mathrm{~cm}$ le long de son axe.

Le dispositif expérimental comprend :

a) Une des sources persistantes type T.301/B/M$22 / 0$ ou T.301/G/M-22/0, fabriquées par Saunders Roe and Nuclear Entreprises Ltd, cylindre creux en verre, recouvert intérieurement de sulfure de zinc activé par environ $2 \mathrm{~cm}^{3}$ de tritium à la pression de $300 \mathrm{~mm}$ de $\mathrm{Hg}$. b) Un photomultiplicateur EMR ASGOP 541 A-05-M 14.

c) Un monochromateur Bausch and Lomb 3386-25.

Les sources phosphorescentes sont placées contre la fente d'entrée du monochromateur, la fenêtre du photomultiplicateur étant à proximité de la fente de sortie.

Après avoir conservé la source « bleue » et la source « verte » pendant 48 heures dans l'obscurité, nous avons enregistré leurs spectres. Ensuite, les deux sources ayant été exposées $1 / 2$ heure au jour et $1 / 4$ d'heure à la lumière d'une lampe à filament de tungstène, nous avons de nouveau enregistré les spectres. Aucune variation due à l'illumination préalable dépassant la marge des erreurs de mesure $(4 \%)$ n'a été observée. A la suite de ce résultat, nous avons laissé reposer les sources pendant 24 heures dans l'osbcurité et effectué un examen en lumière totale, en les disposant contre la fenêtre du photomultiplicateur. Nous avons, ensuite, renouvelé la mesure après la même illumination que précédemment. Aucune variation d'intensité dépassant les erreurs de mesure (1\%) n'a cette fois été décelée, ce qui nous permet de conclure à la haute qualité de ces sources comme étalons secondaires.

Dans le but d'effectuer la calibration des plaques et des films photographiques, nous avons réalisé un petit ensemble fort compact, très facile à transporter et à 
utiliser. Ce dispositif représenté schématiquement ciaprès (fig. 1) comprend :

- une des sources mentionnées précédemment,

- une échelle dégradée Hilger comprenant 6 échelons, dont les densités respectives sont : 0,208 ; 0,$409 ; 0,616 ; 0,807 ; 1,009 ; 1,208$,

- une série de lentilles convergentes,

- un obturateur d'appareil photographique,

- un support mécanique pour l'ensemble.

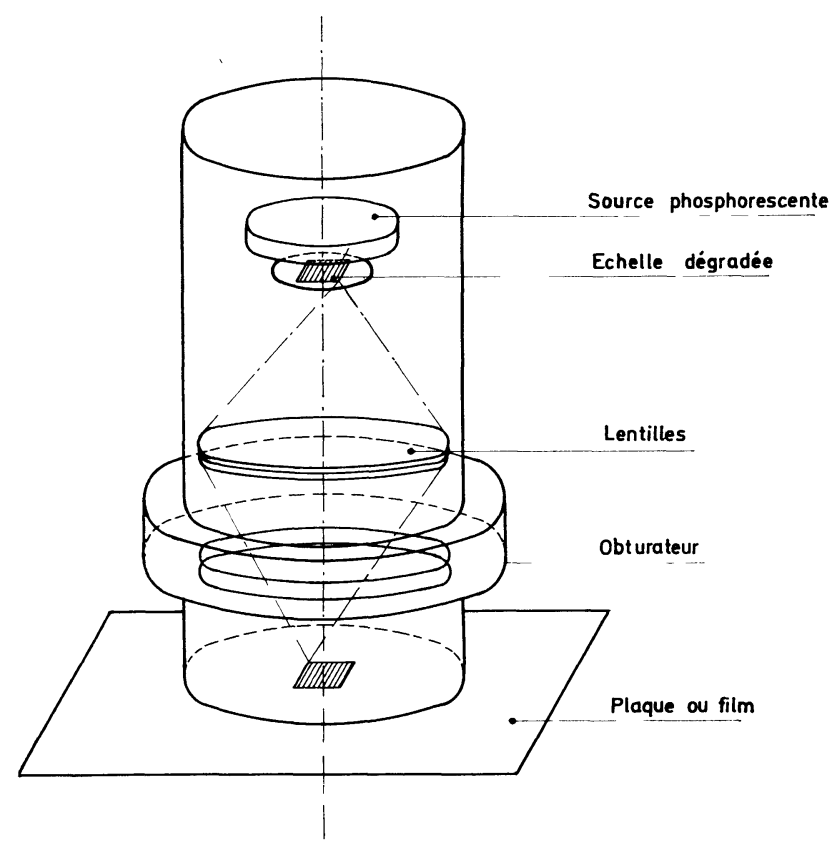

FIG. 1.

La lentille forme sur la plaque une image de l'échelle dégradée éclairée par la source. La répartition de l'intensité en unités absolues $\left(\mathrm{ergs} / \mathrm{cm}^{2}\right.$ ) dans cette image est facile à étudier au moyen d'un photomultiplicateur. Étant donné la période de l'élément excitateur (12,6 ans), cette mesure reste valable pendant un temps suffisamment long.

Pour calibrer une plaque ou un film, il suffit de poser le dispositif sur la plaque, d'armer l'obturateur et de déclencher. Le temps de pose est essentiellement fonction de l'intensité de la source phosphorescente, intensité qui peut varier dans une large gamme, compte tenu de la demande de l'expérimentateur. La photo- graphie ci-jointe ( fig. 2) donne une idée de l'encombrement du prototype réalisé, ainsi qu'un exemple ( fig. 3) de résultat obtenu sur film Agfa Isopan Record.

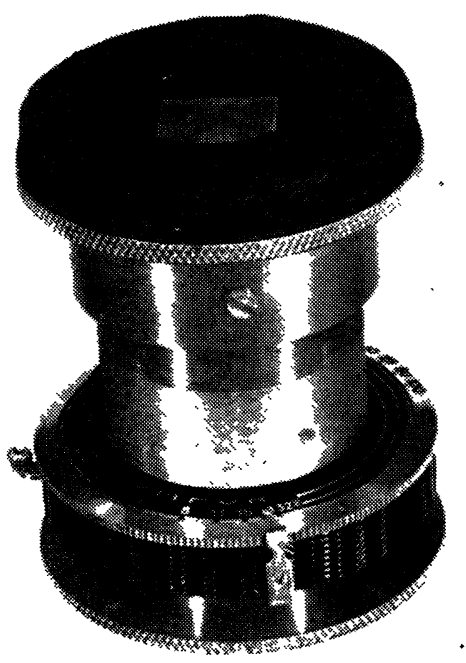

FIG. 2.

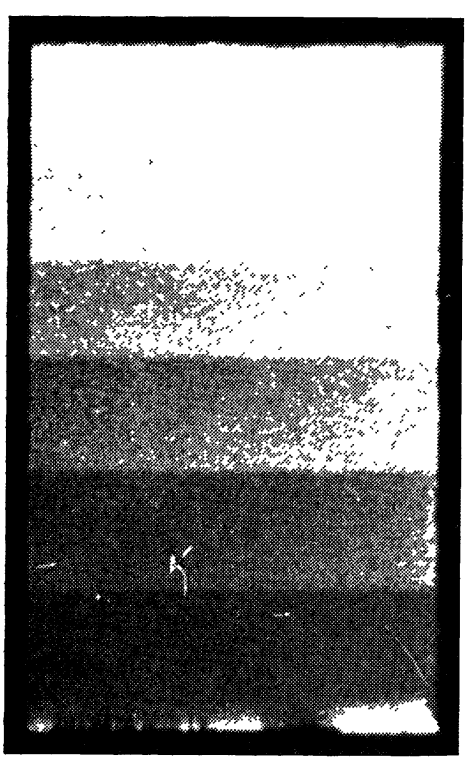

FIG. 3.

\section{BIBLIOGRAPHIE}

[1] Hunten (Donald M.), Space Science Reviews, 1967, 6, 493 . 